\title{
Influence of the heating rate on the critical Marangoni number of oscillatory thermocapillary convection in the floating half zone ${ }^{\dagger}$
}

\author{
LI Kai, TANG ZeMei \& HU WenRui* \\ National Microgravity Laboratory, Institute of Mechanics, Chinese Academy of Sciences, Beijing 100190, China
}

Received October 20, 2011; accepted November 11, 2011; published online December 15, 2011

\begin{abstract}
In the present study, numerical simulations were conducted on thermocapillary convection in floating half zones of $5 \mathrm{cSt}$ silicone oil of different scales in comparison with the experimental studies in the microgravity conditions. The effect of heating rate on the marginal instability boundaries is indicated as a possible explanation for the significant quantitative discrepancies between the experimental results in the terrestrial conditions and in the microgravity conditions.
\end{abstract}

thermocapillary convection, flow instability, finite element methods

PACS number(s): 47.55.nb, 47.20.Ky, 47.11.Fg

Citation: $\quad \mathrm{Li} \mathrm{K}$, Tang Z M, Hu W R. Influence of the heating rate on the critical Marangoni number of oscillatory thermocapillary convection in the floating half zone. Sci China-Phys Mech Astron, 2012, 55: 102-107, doi: 10.1007/s11433-011-4569-5

In fluid systems with interface in the microgravity conditions, capillary effects in comparison with gravity effects play a dominant role. Among them, thermocapillary convection driven by the surface-tension gradient along the interface becomes one of the fundamental subjects in the microgravity fluid physics and the space fluid/heat management [1]. A floating half zone consisting of a liquid column confined between differentially heated solid rods (see Figure 1) was initially introduced to mimic half of the floating zone technique for the interests of space materials science, and it now becomes one of the typical models for the investigation of the principles of thermocapillary convection. Originated by Chun and Wuest [2,3] and Schwabe et al. [4,5] respectively, extensive theoretical studies, e.g., linear instability analyses [6-11], energy stability analyses $[12,13]$ and direct numerical simulations [14-16], have shown that with the increasing applied temperature difference between the supporting rods, thermocapillary convec-

*Corresponding author (email: wrhu@imech.ac.cn)

$\uparrow$ Contributed by HU WenRui (CAS academician) tion transfers from axisymmetric stationary convection to oscillatory convection in the floating half zone of large Prandtl number $(\operatorname{Pr} \gg 1)$. On the other hand, due to the scarce space experiment opportunities, most of the experimental studies available were conducted terrestrially. The relative importance of the gravity effects to the capillary effects is scaled by the static Bond number $\left(B_{0}=\rho g d^{2} / \sigma\right)$ or the dynamic Bond number ( $B_{d}=\rho g \beta d^{2} /\left|\sigma_{T}^{\prime}\right|$ ), where $\rho$ is the fluid density, $g$ the earth gravity level, $d$ the diameter of the floating half zone, $\beta$ the thermal expansion coefficient, $\sigma$ the surface-tension and $\sigma_{T}^{\prime}$ the temperature gradient of the surface-tension. Therefore, the geometrical scale of the floating half zone in the terrestrial conditions was reduced to several millimeters in diameter to make the capillary effects dominant. However, the most important space experiments on thermocapillary convection in the floating half zone onboard the Spacelab D-2 [17,18] predicted that the critical Marangoni numbers determined are about one order of magnitude larger than those in the terrestrial experiments (Table 1). Moreover, the critical Marangoni numbers increase with the increasing diameter of the cylindrical floating 


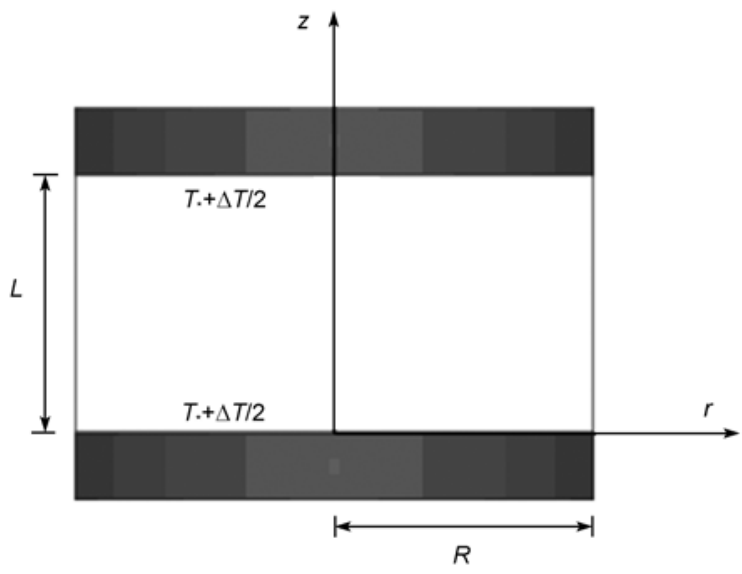

Figure 1 Schematic diagram of the floating half zone.

Table 1 Experimental results of D-2 mission on board the Spacelab after [18]

\begin{tabular}{cccccc}
\hline $\begin{array}{c}\text { Diameter } \\
(\mathrm{mm})\end{array}$ & $\begin{array}{c}\text { Height } \\
(\mathrm{mm})\end{array}$ & $A$ & $\Delta T\left({ }^{\circ} \mathrm{C}\right)$ & $(M a)_{\mathrm{c}}$ & $f_{\mathrm{c}}(\mathrm{Hz})$ \\
\hline 30.0 & 30.0 & 1.0 & 10.1 & 68000 & $\cdots$ \\
30.0 & 45.0 & 1.5 & 17.9 & 180000 & $\ldots$ \\
30.0 & 60.0 & 2.0 & 15.9 & 213000 & 0.32 \\
45.0 & 33.75 & 0.8 & 15.2 & 114000 & 0.2 \\
45.0 & 45.0 & 1.0 & 12.0 & 120000 & 0.14 \\
45.0 & 67.5 & 1.5 & 12.3 & 185000 & 0.21 \\
60.0 & 45.0 & 0.8 & 16.5 & 166000 & 0.12 \\
60.0 & 60.0 & 1.0 & 17.2 & 230000 & 0.12 \\
\hline
\end{tabular}

half zone of the same aspect ratio. It may be expected that the dimensional scale is an important critical parameter for the oscillatory thermocapillary convection in the floating half zone of large scales in the microgravity conditions.

On the other hand, the applied temperature difference is usually increased at a certain heating rate in the experiment procedure until the oscillatory convection occurs. Generally, a low heating rate is adopted in the terrestrial experiments, and the heating procedure can be considered as quasi-steady; a much higher heating rate is required in the short-term space experiments such as those conducted in the Spacelab D-2. In our previous terrestrial experimental research [19], the prominent influence of heating rate on the experimental determination of the critical Marangoni was studied. The results show that the larger the heating rate, the higher the critical Marangoni number for the floating half zone with the fixed geometric parameter. This study provided an important clue to explaining the dispersion of the terrestrial and space experimental results. However, the diameter of the floating half zone ranges from $2 \mathrm{~mm}$ to $5.5 \mathrm{~mm}$ due to the limitation of the terrestrial conditions, and the gravity effects are unlikely to be totally excluded.

This paper numerically studied the influence of the heating rate on the determination of the critical Marangoni number of thermocapillary convection in floating half zones of diameters ranging from $3 \mathrm{~mm}$ to $45 \mathrm{~mm}$ in the microgravity conditions. As a possible explanation for the significant quantitative discrepancy mentioned above, we indicate that the heating rate is a critical parameter of the oscillatory thermocapillary convection in the floating half zone.

\section{Physical and mathematical model}

A schematic diagram of the floating half zone confined between two co-axial copper rods is shown in Figure 1. The applied temperature difference $(\Delta T)$ between the rods can be either fixed or time-dependent. The non-dimensional governing equations for the thermocapillary convection in the floating half zone with the Boussinesq approximation are written as:

$$
\begin{gathered}
\frac{\partial \boldsymbol{\omega}}{\partial \tau}+\boldsymbol{U} \cdot \nabla \boldsymbol{\omega}-\boldsymbol{\omega} \cdot \nabla \boldsymbol{U}=\frac{\operatorname{Pr}}{M a^{*}}\left[\nabla^{2} \omega+\frac{\operatorname{PrGr}^{*}}{M a^{*}} \nabla \times(T \hat{\boldsymbol{e}})\right], \\
\frac{\partial \Theta}{\partial \tau}+\boldsymbol{U} \cdot \nabla \Theta=\frac{1}{M a^{*}} \nabla^{2} \Theta .
\end{gathered}
$$

In the cylindrical coordinates $(\xi, \theta, \zeta)$ the non-dimensional vorticity vector $\boldsymbol{\omega}=\left(\omega_{\xi}, \omega_{\theta}, \omega_{\zeta}\right)$ and stream function vector $\boldsymbol{\psi}=\left(\psi_{\xi}, \psi_{\theta}, \psi_{\zeta}\right)$ are defined as:

$$
\begin{gathered}
\nabla \times \boldsymbol{\psi}=\boldsymbol{U}, \\
\nabla \times \nabla \times \boldsymbol{\psi}=\boldsymbol{\omega},
\end{gathered}
$$

where $\hat{\boldsymbol{e}}$ is the unit vector of gravity acceleration and $\boldsymbol{U}=$ $(u, v, w)$ the non-dimensional velocity vector. The length, velocity, pressure and time are scaled by

$$
L, \quad U_{0}=\left|\sigma_{T}^{\prime}\right| \Delta T_{*} / \rho v, \quad \rho U_{0}^{2}
$$

and $L / U_{0}$ respectively, and the temperature is scaled by $\Delta T_{*}=T_{*}-T_{0}$, where $L$ is the height of the floating half zone, $T_{0}$ is the constant temperature at the lower rod and $T_{*}$ is the maximum temperature at the upper rod in the heating process. The non-dimensional parameters are defined as:

$$
\begin{gathered}
M a^{*}=U_{0} L / \kappa, \quad \operatorname{Pr}^{*}=v / \kappa, \\
G r^{*}=g \beta \Delta T L^{3} / v \kappa,
\end{gathered}
$$

where $v$ is the kinetic viscosity and $\kappa$ the thermal diffusion coefficient of the liquid respectively. Note that for a specific applied temperature difference $\Delta T, M a=M a^{*} \Delta T / \Delta T_{*}$ was defined accordingly.

The free surface is described as $\xi=r / L=R(\zeta)$ with $R(0)=$ $R(1)=0.5 \mathrm{Ar}$, where $A r=L / 2 R_{0}$ is the aspect ratio of floating half zone. The non-dimensional boundary conditions can be expressed as follows:

When $\zeta=0$ and $\zeta=1$ : 


$$
\begin{gathered}
\psi_{\xi}=\psi_{\theta}=0, \quad \frac{\partial \psi_{\zeta}}{\partial \zeta}=0, \\
\omega_{\xi}=-\frac{\partial v}{\partial \zeta}, \quad \omega_{\theta}=\frac{\partial u}{\partial \zeta}, \quad \omega_{\zeta}=0, \\
\Theta(\xi, \theta, 0, \tau)=0, \quad \Theta(\xi, \theta, 1, \tau)=f(\tau),
\end{gathered}
$$

when $\xi=R(\zeta)$ :

$$
\begin{aligned}
\psi_{\xi}=\psi_{\theta}=0, \quad \nabla \cdot \psi=0 \\
=\frac{1}{\xi} \frac{\partial}{\partial \theta}\left(\frac{1}{\xi} \frac{\partial \xi \psi_{\theta}}{\partial \xi}-\frac{1}{\xi} \frac{\partial \psi_{\zeta}}{\partial \theta}\right)-\frac{\partial}{\partial \zeta}\left(\frac{\partial \psi_{\xi}}{\partial \zeta}-\frac{\partial \psi_{\zeta}}{\partial \xi}\right), \\
\omega_{\theta}=\frac{2 R}{\left(1-R^{2}\right)} \\
\times\left[\frac{\partial}{\partial \theta}\left(\frac{1}{\xi} \frac{\partial \xi_{\theta}}{\partial \theta}-\frac{\partial \psi_{\zeta}}{\partial \zeta}\right)-\frac{\partial}{\partial \zeta}\left(\frac{1}{\zeta} \frac{\partial \xi \psi_{\theta}}{\partial \xi}-\frac{1}{\zeta} \frac{\partial \psi_{\xi}}{\partial \theta}\right)\right] \\
+2 \frac{\partial}{\partial \zeta}\left(\frac{1}{\xi} \frac{\partial \xi_{\zeta}}{\partial \theta}-\frac{\partial \psi_{\theta}}{\partial \zeta}\right)-\frac{\left(1+R^{2}\right)}{\left(1-R^{2}\right)} \frac{\partial \Theta}{\partial s}, \\
\quad \omega_{\zeta}=\frac{\left(1+R^{2}\right)^{\frac{1}{2}}}{\xi} \frac{\partial \Theta}{\partial \theta}+2 \frac{\partial}{\partial \xi}\left(\frac{\partial \psi_{\xi}}{\partial \zeta}-\frac{\partial \psi_{\zeta}}{\partial \xi}\right) \\
-R\left[\omega_{\xi}+2 \frac{\partial}{\partial \zeta}\left(\frac{\partial \psi_{\xi}}{\partial \zeta}-\frac{\partial \psi_{\zeta}}{\partial \xi}\right)\right] \\
\frac{\partial \Theta}{\partial n}=0,
\end{aligned}
$$

where $n$ and $s$ stand respectively for the unit vector in the normal direction of the free surface and perpendicular to both $n$ and the azimuthal direction of the free surface, and $f(\tau)$ is given by

$$
f(\tau)= \begin{cases}\alpha \tau, & \tau<T_{*} / \alpha \Delta T_{*}, \\ T_{*} / \Delta T_{*}, & \tau \geqslant T_{*} / \alpha \Delta T_{*},\end{cases}
$$

where the non-dimensional heating rate $\alpha$ is constant. The floating half zone is initially set as isothermal without convection.

\section{Procedure and code validation}

In the present study, the hybrid finite element method of fractional steps was adopted to solve the governing equations with the corresponding initial and boundary conditions. The finite element method (FEM) is widely adopted in the computational fluid dynamics due to its stability, simplicity and flexibility in the handling of the complex computation domain. Under the hybrid finite element method of frac- tional steps, a characteristic line method and a finite element method are respectively applied to the convection term to account for the strong non-linear effect and the diffusion operator. It was firstly suggested by Yanenko [20]. Wu [21] adopted the hybrid finite element method of fractional steps for the numerical modeling of aquatic environment and provided the corresponding detailed stability analysis.

For the numerical studies reported in the following sections, the computation domain is divided into 20430 tetrahedron elements (see Figure 2) totally with 1362 tetrahedron elements in each horizontal mesh layer. Note that an increasing mesh density in the vicinity of the computation domain boundary was adopted, and no node is assigned at the vertical axis of the cylindrical coordinate system to avoid singularities. The code has been validated against the experimental and numerical studies on the floating half zone of $5 \mathrm{cSt}$ silicone oil [17]. Figure 3 shows the comparison of
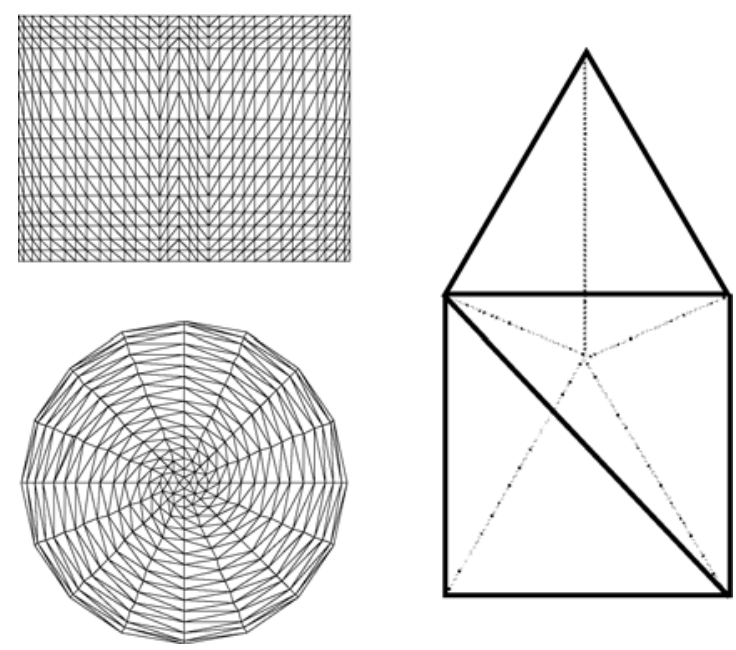

Figure 2 Schematic diagrams of the tetrahedron elements distribution in the $(\xi, \theta, \zeta)$ directions.

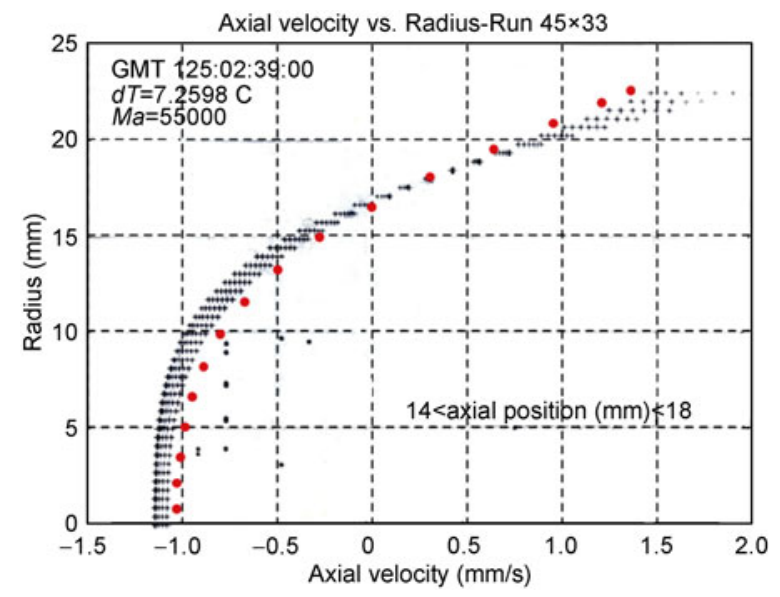

Figure 3 (Color online) Comparison of the axial velocities in the midplane of the floating half zone of $5 \mathrm{cSt}$ silicone oil at $M a=55000$. * the experimental measurement, + the numerical simulation in Figure 10 of ref. [17], and $\bullet$ the numerical simulation by the present code. 
the axial velocities in the midplane of the floating half zone at $M a=55000$ by the experimental measurement and numerical simulation [17] and the present numerical simulation. The results are in good agreement, which indicates the reliability of the present numerical simulation for the cases of large Prandtl number.

\section{Threshold for oscillatory convection}

The most sensitive feature of the onset of oscillatory thermocapillary convection in a floating half zone is the evolution of the azimuthal velocities. When the applied temperature difference exceeds a threshold, the azimuthal velocities grow from zero and start to oscillate (not shown here). The threshold for the onset of oscillatory convection in the present study is defined based on the non-axisymmetric degree of the azimuthal velocities:

$$
\delta_{\mathrm{v}} \geqslant \frac{v_{\max }-v_{\min }}{U_{\max }}=2 \times 10^{-2},
$$

where $v_{\max }$ and $v_{\min }$ are respectively the maximum and minimum of the azimuthal velocities on the free surface at the horizontal cross-section of $\xi=0.55$, and $U_{\max }$ is the maximum velocity of the convection in the floating half zone.

\section{Results and discussion}

For a better understanding of the quantitative discrepancy between the terrestrial experimental results on floating half zones of several millimeters in diameter and the space experimental results on floating half zones of several centimeters in diameter [18], numerical simulations were conducted on the floating half zone of $5 \mathrm{cSt}$ silicone oil $(\mathrm{Pr}=73$, $A r=1.0$ ) in the microgravity conditions (Table 2 shows the corresponding thermophysical properties). With the unitary aspect ratio and cylindrical configuration, the diameters of the floating half zones were selected as 45, 30, 20, 10 and 3 $\mathrm{mm}$ respectively, which approximately cover the scales of the experimental apparatus in the terrestrial conditions and in the microgravity conditions. Figure 4 shows the heating-rate dependent critical Marangoni number determined for floating half zones of different scales. Generally speaking, for floating half zones of different scales heated at the same heating rate, the critical Marangoni number increases with the increasing scale. On the other hand, the tendencies of the marginal instability boundaries are relatively complex for the intermediate floating half zones of $d=20$ and $10 \mathrm{~mm}$, especially in the range of heating rates less than $0.003 \mathrm{~K} / \mathrm{s}$. Note that such a low heating rate is only practical in a long-term space experimental mission such as in the ISS. For the large floating half zones of $d=45$ and $30 \mathrm{~mm}$, the critical Marangoni number approximately increases linearly with the increasing heating rate. For the small floating half zone of $d=3 \mathrm{~mm}$, the critical Marangoni number nearly keeps constant with the increasing heating rate (see Figure 4). Figure 5(a) shows the evolution of the azimuthal velocities at four monitoring points on the free surface (with a phase shift of $\pi / 2$ ) at the horizontal cross-section of $\xi=0.55$ for the floating half zone of $d=30 \mathrm{~mm}$ with the heating rate of $0.00005 \mathrm{~K} / \mathrm{s}$. The azimuthal velocities grow from zero and start to oscillate when the applied temperature difference exceeds $\Delta T_{\mathrm{c}}=7.612 \mathrm{~K}$. Multiple frequencies (coupled high and low oscillation frequencies) co-exist right after the onset of the oscillatory thermocapillary convection, which can be clearly observed through the corresponding frequency spectrum by FFT transformation (see Figure 5(b)). In the range of high heating rates, the evolution of the monitoring azimuthal velocities exhibits intensive aperiodicity, which reflects the large disturbances on the flow field induced by the rapidly increased applied temperature difference, so does the corresponding frequency spectrum (see Figure 6).

It is worth noting that in the range of the practical heating rates in the short-term space experiments $(0.008-0.013 \mathrm{~K} / \mathrm{s})$ [18], the numerically determined critical temperature dif-

Table 2 Thermophysical properties of $5 \mathrm{cSt}$ silicone oil

\begin{tabular}{cc}
\hline Parameters & Value \\
\hline Kinetic viscosity $v$ & $4.9405 \times 10^{-6} \mathrm{~m}^{2} \mathrm{~s}^{-1}$ \\
Thermal expansion coefficient $\beta$ & $1.09 \times 10^{-3} \mathrm{k}^{-1}$ \\
Density $\rho$ & $0.91 \times 10^{3} \mathrm{~kg} \mathrm{~m}^{-3}$ \\
Thermal conductivity $\kappa$ & $6.6938 \times 10^{-8} \mathrm{~m}^{2} \mathrm{~s}^{-1}$ \\
Surface tension $\sigma$ & $1.97 \times 10^{-2} \mathrm{~N} \mathrm{~m}^{-1}$ \\
Temperature gradient of surface tension $\sigma$ & $-6.37 \times 10^{-5} \mathrm{~N} \mathrm{~m}^{-1} \mathrm{k}^{-1}$ \\
\hline
\end{tabular}

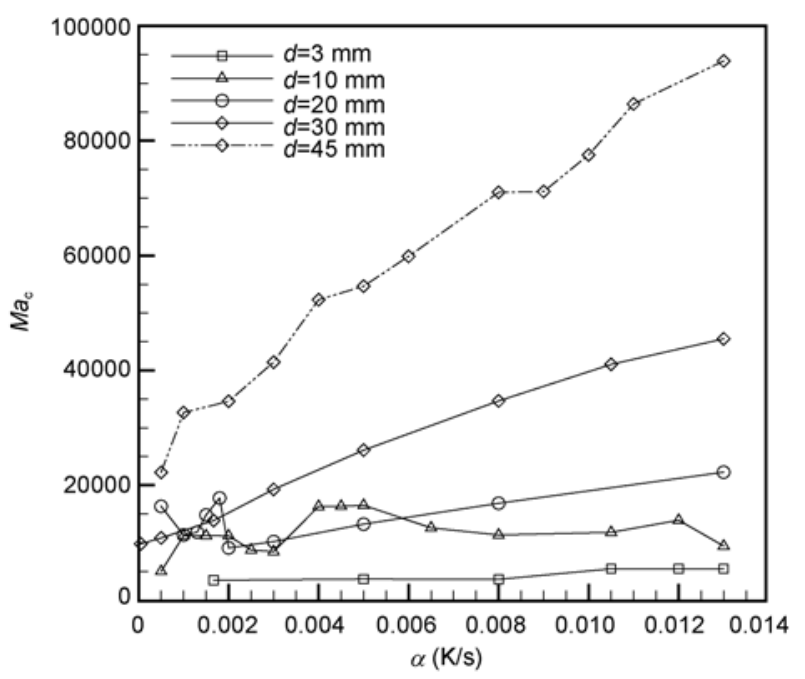

Figure 4 Dependency of the critical Marangoni number on the heating rate for floating half zones ( $5 \mathrm{cSt}$ silicone oil) of different scales with $V / V_{0}$ $=1.0$ in the microgravity conditions. 

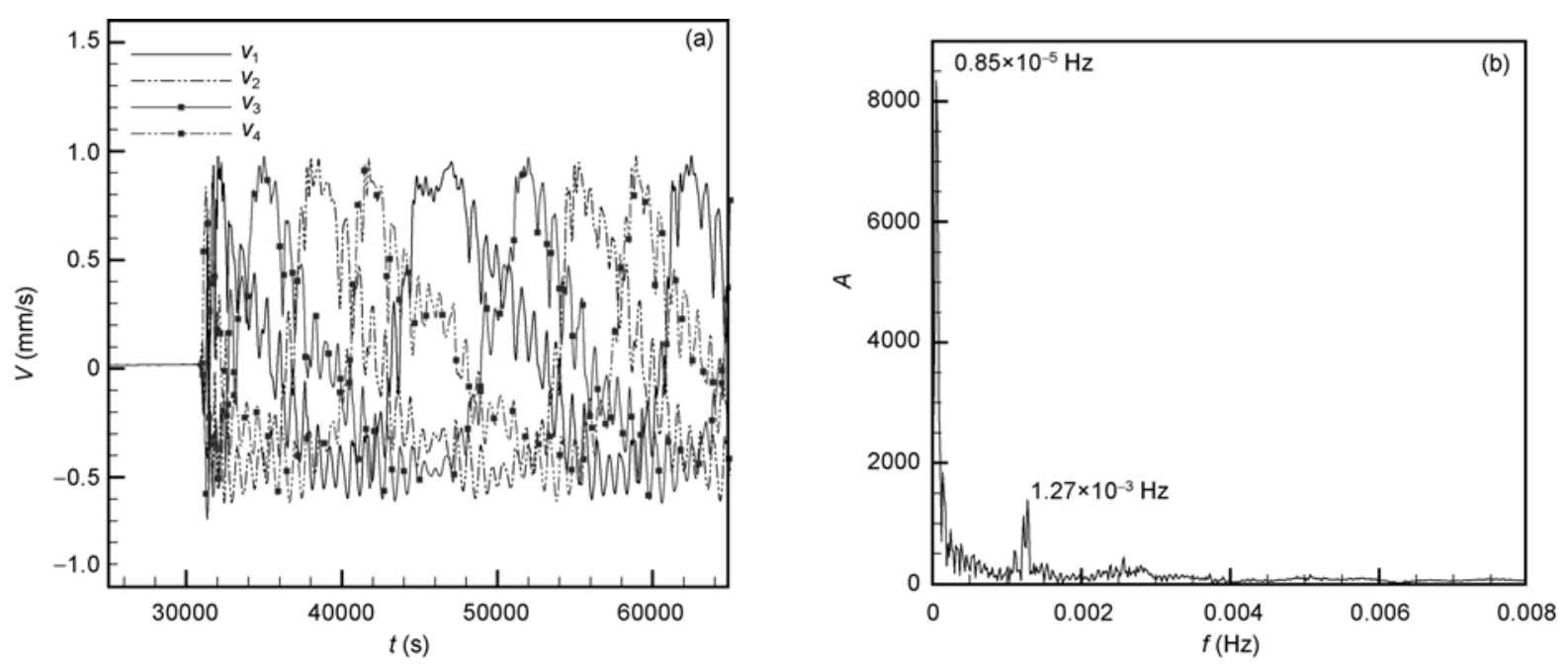

Figure 5 For the floating half zone of $d=30 \mathrm{~mm}$ with the heating rate of $0.00005 \mathrm{~K} / \mathrm{s}$ : (a) evolution of the azimuthal velocities at the monitoring points and (b) the corresponding frequency spectrum by FFT.
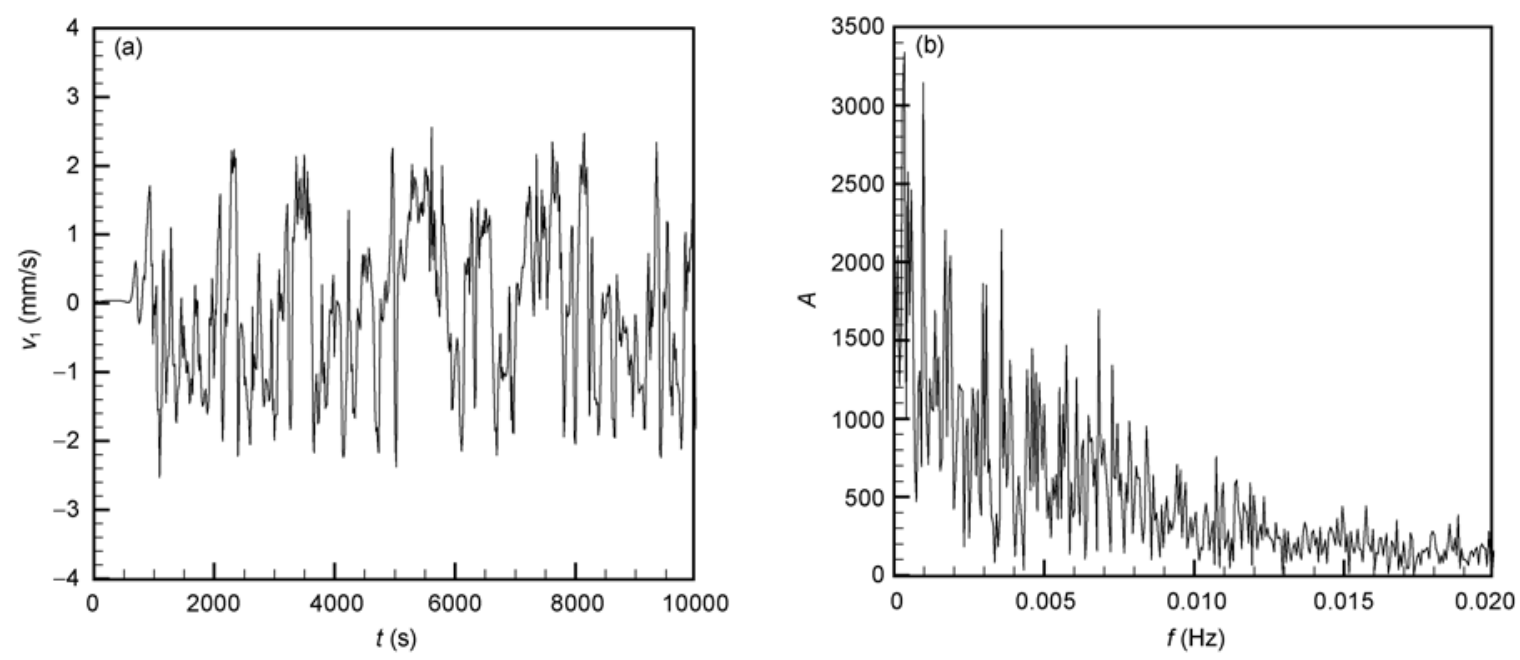

Figure 6 For the floating half zone of $d=30 \mathrm{~mm}$ with the heating rate of $0.013 \mathrm{~K} / \mathrm{s}$ : (a) evolution of the azimuthal velocities at the monitoring points and (b) the corresponding frequency spectrum by FFT.

ferences for the floating half zone of $d=30 \mathrm{~mm}$ range from $\Delta T_{\mathrm{c}}=5.8 \mathrm{~K} \quad\left(M a_{\mathrm{c}}=34763\right)$ to $\Delta T_{\mathrm{c}}=7.6 \mathrm{~K} \quad\left(M a_{\mathrm{c}}=45000\right)$, which are quite close to the space experimental result, $\Delta T_{\mathrm{c}}=10.1 \mathrm{~K}\left(M a_{\mathrm{c}}=68000\right)$ [18]. On the other hand, the critical Marangoni number for the small floating half zone of $d=3 \mathrm{~mm}$ at a low heating rate is much smaller, e.g., $M a_{\mathrm{c}}=$ 3436 at the heating rate of $0.0017 \mathrm{~K} / \mathrm{s}$.

Moreover, different from the numerical studies where the applied temperature difference is directly imposed at the $\mathrm{rod} / \mathrm{melt}$ interfaces, in the practical experiment procedure, the heater supplies heat to the rod, and a part of the heat is transferred from the heater via a copper rod to the liquid column through convection and conduction to establish the temperature field; the rest leads to the rise in the rod temperature. More heat would cause the rise of rod temperature if the heating rate is high, and a relatively higher applied temperature difference is measured when the oscillation occurs, i.e., a time lag exists between the applied temperature difference and the full development of the responding thermal disturbances in the whole liquid column. It becomes significant for the floating half zone of several centimeters in diameter with a high heating rate.

The above phenomena may partially explain the significant discrepancy of the critical Marangoni number in magnitude between the terrestrial experimental results and the space experimental results, i.e., the heating rate is an important critical parameter in critical Marangoni number determination for the oscillatory thermocapillary convection in the floating half zone, especially in the practical experiment procedure. Other factors, such as the more significant effect of boundary layers formed at the supporting rods in floating half zones of several millimeters in diameter in comparison with floating half zones of several centimeters in diameter and the gravity effect including the free-surface deformation 
[22] in floating half zones of several millimeters in diameter in the terrestrial conditions, may also possibly contribute to the discrepancy.

Recently, an excellent long-term space experiment on the floating half zone of $30 \mathrm{~mm}$ in diameter was conducted in the "KIBO" JEM in ISS with a low heating rate. They predicted nearly the same critical Marangoni number as that for the floating half zone of a small diameter contrary to the experimental predictions in the Spacelab D-2. This phenomenon experimentally verified the important role of the heating rate in the thermocapillary convection instability as indicated in the present study.

\section{Conclusions}

In the present study, the influence of heating rate on the marginal instability boundaries for floating half zones scaled from $45 \mathrm{~mm}$ to $3 \mathrm{~mm}$ were numerically studied in the microgravity conditions. For floating half zones of large scales, the tendency of the marginal instability boundary is relatively complex in the range of very low heating rates while it nearly increases linearly with the increasing heating rate. For floating half zones of different scales heated at the same heating rate, the critical Marangoni number increases with the increasing scale. Within the range of the practical heating rates in the space experiments, i.e., $0.008-0.013 \mathrm{~K} / \mathrm{s}$, the critical Marangoni numbers are close to the space experimental results. Therefore, this phenomenon may be one of the possible interpretations on the significant discrepancy of the critical Marangoni number in magnitude between the terrestrial experimental results and the space experimental results.

This work was supported by the National Natural Science Foundation of China (Grant Nos. 11032011 and 10872202).

1 Hu W R, Tang Z M, Li K. Thermocapillary flow in floating zones. Appl Mech Rev, 2008, 61: 010803-15

2 Chun $\mathrm{Ch} \mathrm{H}$, Wuest W. A micro-gravity simulation of the Marangoni convection. Acta Astronaut, 1978, 5: 681-686

3 Chun $\mathrm{Ch} \mathrm{H}$, Wuest W. Experiments on the transition from the steady to the oscillatory Marangoni-convection of a floating zone under reduced gravity effect. Acta Astronaut, 1979, 6: 1073-1082
4 Schwabe D, Scharmann A, Preisser F, et al. Experiments on surface tension driven flow in floating zone melting. J Cryst Growth, 1978, 43: 305-312

5 Schwabe D, Scharmann A. Some evidence for the existence and magnitude of a critical Marangoni number for the onset of oscillatory flow in crystal growth melts. J Cryst Growth, 1979, 46: 125-131

6 Smith M K, Davis S H. Instability of dynamic thermocapillary liquid layer, Part I: Convective instability. J Fluid Mech, 1983, 132: 119-144

7 Neitzel G P, Chang K T, Jankowski D F, et al. Linear stability of thermocapillary convection in a model of the floating zone crystal growth process. AIAA Paper, 1992, AIAA-92-0604

8 Wanschura M, Shevtsova V M, Kuhlmann H C, et al. Convective instability mechanisms in thermocapillary floating half zone. Phys Fluids, 1995, 7: 912-925

9 Chen G, Lizee A, Roux B J. Bifurcation analysis of the thermocapillary convection in cylindrical floating half zone. J Cryst Growth, 1997, 180: 638-647

10 Chen Q S, Hu W R. Instability of floating half zone volume on instability of floating half zone convection. Int J Heat Mass Transfer, 1998, 41: 825-837

11 Chen Q S, Hu W R, Prasade V. Effect of floating half zone volume on the instability in small Prandtl number half zone. J Cryst Growth, 199, 203: 261-268

12 Kuhlmann H C, Rath H J. Hydrodynamic instabilities in cylindrical thermocapillary floating half zones. J Fluid Mech, 1993, 247: 247274

13 Neitzel G P, Law C C, Jankowski D F, et al. Energy stability of thermocapillary convection in a model of the floating zone crystal growth process. Phys Fluids A, 1991, 3: 2841-2846

14 Savino R, Monti R. Oscillatory Marangoni convection in cylindrical floating half zone. Phys Fluids, 1996, 8: 2906-2922

15 Shevtsova V M, Legros J C. Oscillatory convection motion in deformed floating half zones. Phys Fluids, 1998, 10: 1621-1634

16 Tang Z M, Hu W R, Imaishi N. Two bifurcation transitions of the floating half zone convection in a fat floating half zone of larger $\mathrm{Pr}$ number. Int J Heat Mass Transfer, 2001, 44: 1299-1307

17 Carotenuto L, Castagnolo D, Albanese C, et al. Instability of thermocapillary convection in liquid bridges. Phys Fluids, 1998, 10: 555565

18 Monti R, Albanese C, Carotenuto L, et al. First results from "onset" experiment during Spacelab mission D-2. In: Proceedings of the Norderney Symposium on Scientific Results of the German Spacelab Mission D2. Sahm P R, Keller M H, Schiewe B, eds. 1995. 247-258

19 Xie J C, Hu W R. Influence of heating rate on onset of oscillation in liquid bridge of half floating zone. J Thermal Sci, 1994, 3: 122-125

20 Yanenko N N. The method of fractional steps. Holt M, ed. New York: Springer-Verlag Berlin Heidelberg, 1971

21 Wu J H. A hybrid method of fractional steps with L-stability for numerical modeling of aquatic environments. Adv Hydrodyn, 1985, 1: 27-36

22 Xun B, Li K, Chen P G, et al. Effect of interfacial heat transfer on the onset of oscillatory convection in floating half zone. Int $\mathrm{J}$ Heat Mass Transfer, 2009, 52: 4211-4220 